\title{
Modular Invariance and the Fusion Algebra
}

\author{
Robbert Dijkgraaf and Erik Verlinde ${ }^{1}$ \\ Institute for Theoretical Physics, University of Utrecht, P.O.Box 80.006, 3508 TA Utrecht \\ The Netherlands
}

ABSTRACT. We discuss the relation between modular transformations and the fusion algebra, and explain its proof. It is shown that the existence of off-diagonal modular invariant partition functions imply the existence of a non-trivial automorphism of the fusion algebra. This is illustrated using the $S U(2)$ affine models.

\section{Introduction}

Recently it has become clear that in 2D conformal field theory a central role is played by the fusion rules. They can be expressed as a commutative and associative algebra with integral coefficients carrying information about the operator product algebra of the primary fields [1]. For a given set of fusion rules one can derive in several ways powerful constraints on the central charge and conformal dimensions $[1,2,3,4]$. These constraints are particularly useful for rational CFT's and may help in an eventual classification.

There exists a intriguing relation between the fusion algebra and the modular properties of the one-loop characters: the matrix $S$ describing the behavior under $\tau \rightarrow-1 / \tau$ diagonalizes the algebra. The proof of this fact was indicated in [1] and completed recently by Moore and Seiberg [5] using the socalled 'pentagon identity'. The relation between $S$ and the fusion algebra has many useful applications, some of which will be discussed in this paper.

In the first part we will, after describing the definition of the fusion algebra, explain its connection with modular transformations. As a corollary we find that the matrix $S$ is symmetric and unitary. To show how some of these things work in practice we discuss an ex- ample: the 4 state Potts model. In the second part we study the implications of our results for the structure of modular invariant partition functions. We will find that off-diagonal invariants can only exist when the fusion algebra allows some non-trivial automorphism. This is illustrated with the off-diagonal combinations of the $A_{1}^{(k)}$ affine characters. This part was motivated by recent work of Brustein, Yankielowicz, and Zuber on factorization of the operator algebra [6].

\section{The Fusion Algebra}

Let us begin with some basic definitions and assumptions. We consider conformal field theories with a left and right chiral symmetry algebra that consist of integer spin generators and both contain the Virasoro algebra at some value $c$ of the central charge. For convenience we will take the left and right algebra to be isomorphica restriction that is quite reasonable in the context of critical phenomena, but excludes for example heterotic string models. The field content is organised in representations of the full symmetry algebra of the model, that possibly include a discrete symmetry group. A CFT is called compact if its spectrum of irreducible representations is discrete, and rational if it is finite. We restrict

1 address after 1 October 1988: Institute for Advanced Study, Princeton NJ 08540, USA 
ourselves to the latter, although much of the following applies to the general case.

The representations can be decomposed in tensor products of the irreducible representations $\left[\phi_{i}\right]$ of the chiral algebra. Thus the Hilbert space will have the structure

$$
\mathcal{H}=\bigoplus_{(i, \bar{i})}\left[\phi_{i}\right] \otimes\left[\bar{\phi}_{\bar{i}}\right]
$$

where the indices $i$ and $\bar{i}$ range over the same set of $N$ labels. The primary operators corresponding to the heighest weight representation $\left[\phi_{i}\right] \otimes\left[\bar{\phi}_{\bar{i}}\right]$, which in general form a multiplet, will be collectively denoted as $\Phi_{i \bar{i}}(z, \bar{z})$. Note that in $(2.1)$ a pair $(i, \bar{i})$ is not allowed to occur with multiplicity larger than one. The rational behind this is that there will always be some quantum number that distinguishes between two non-identical operators.

The one-loop partition function is given by a trace over the Hilbert space $\mathcal{H}$ and decomposes as

$$
Z(\tau, \bar{\tau})=\sum_{(i, \bar{i})} \chi_{i}(\tau) \bar{\chi}_{\bar{i}}(\bar{\tau})
$$

with the characters $\chi_{i}$ defined by $\chi_{i}=\operatorname{tr}_{\left[\phi_{i}\right]}\left(q^{L_{0}-\frac{c}{24}}\right)$. It is well possible that the characters of two different representations $\left[\phi_{i}\right]$ have the same $q$-expansion. We will see some examples of this in the subsequent sections. An important physical consistency requirement imposed on $Z$ is invariance under the modular group, generated by $T: \tau \rightarrow \tau+1$ and $S: \tau \rightarrow-1 / \tau$. In order to obtain a better understanding of the implications of modular invariance, we will first consider the analytical structure of the correlation functions of the theory.

Conformal invariance allows one to consider correlation functions on arbitrary Riemann surfaces. These multi-point functions are known to possess a particular analytic structure: a $n$-point function on a genus $g$ surface can be written as a sum of terms, that each factorize in a holomorphic times an anti-holomorphic expression in the $n+3 g-3$ complex moduli of the $n$-punctured surface [7]. Two familiar examples are the one-loop partition function [8] and the 4-point function on the sphere [9] of the minimal models, where the holomorphic blocks are the characters resp. conformal blocks of the Virasoro algebra. The emergence of this structure of the correlation functions can be understood as follows.

We can choose on the Riemann surface $2 n+3 g-3$ non-intersecting, primitive homotopy cycles, $n$ of which encircle the punctures. In the neighbourhoud of these cycles we can define locally a time-direction, which can be used to analyze the spectrum in these channels, and, in particular allows us to project on a representation $\left[\phi_{i}\right] \otimes\left[\bar{\phi}_{i}\right]$. The choice of cycles divides the surface in $n+$ $2 g-2$ 'pants', that couple the three adjacent channels. Assuming that all the three-point couplings factorizewhich is true for many CFT's such as the Ising model- it is sufficient to project onto representations in the channels to obtain a factorized amplitude. However, in general the three-point couplings do not factorize, and we should further project on specific type of couplings.

This is best demonstrated with the aid of an example. Consider the $S U(3)$ WZW-model for high enough level $k$. Then we have a representation $\left[\phi_{8}\right] \otimes\left[\bar{\phi}_{8}\right]$ corresponding to the $8 \times 8$-dimensional adjoint representation of the zero mode part of the $A_{2}^{(1)} \times A_{2}^{(1)}$ current algebra. The corresponding primary fields are denoted as $\Phi^{a \bar{a}}(a, \bar{a}=1, \ldots, 8)$ where we now explicitly indicated the multiplet structure. It is well-known that the space of Clebsch-Gordon coefficients for $\mathbf{8} \times \mathbf{8}=\mathbf{8}$ is two-dimensional. One usually chooses as independent couplings the completely symmetric resp. antisymmetic forms $d_{a b c}$ and $f_{a b c}$. In terms of these the most general form of the correlator of three of these primary fields 
reads

$$
\left\langle\Phi^{a \bar{a}}(0) \Phi^{b \bar{b}}(1) \Phi^{\bar{c} \bar{c}}(\infty)\right\rangle=C^{(d)} d_{a b c} d_{\bar{a} \bar{b} \bar{c}}+C^{(f)} f_{a b c} f_{\bar{a} \bar{b} \bar{c}}
$$

Note that a term of the form $d \cdot f$ is excluded by the requirement of permutation symmetry ( $=$ 'modular invariance') of the three-point function.

As this example shows, it is possible that three representations have more than one independent couplings. In fact the $S U(3)$ example is a bit misleading, since it suggests that multiple couplings only occur when the ground state of the representation is degenerate. However, orbifold and coset models provide examples to the contrary [10].

Summarizing, the correlation functions decompose into holomorphic blocks after projection on channels and couplings. These blocks can be labelled by a $\varphi^{3}$. diagram, with propagators

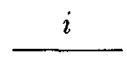

and vertices

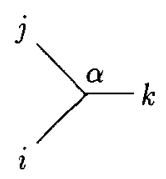

where we denoted the type of coupling by the index $\alpha=1, \ldots, N_{i j k}$. For complex representations the propagator carries an orientation. We note however that the correspondence with $\varphi^{3}$-diagrams is not in all cases unambiguous.

Of course there is no unique marking of the surface. Different choices of channels furnish different bases in the vector space of holomorphic blocks. The completeness of each basis, corresponding to a $\varphi^{3}$-diagram of particular type, is a consequence of the consistency of the model, and is of fundamental importance in all further considerations.
We can now introduce the fusion algebra. It is defined as the associative algebra

$$
\phi_{i} \times \phi_{j}=N_{i j}{ }^{k} \phi_{k}
$$

where the non-negative integer $N_{i j k}$ is the number of independent couplings of the type $(i j k)$. (Indices are raised and lowered by the conjugation matrix $C_{i j}=\delta_{i j}$.) Closure of the chiral algebra, and the fact that $\left[\phi_{i}\right]$ is a representation imply the existence of an identity element: $1 \times \phi_{i}=\phi_{i}$.

The associativity of the fusion algebra follows from the fact that both the $s$ - and $t$-channel conformal blocks<smiles>[Y]C([3H])C([3H])I</smiles>
and

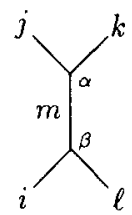

form a complete basis. Since (2.6) is also commutative, it has $N$ irreducible one-dimensional representations $\lambda_{i}^{(n)}$

$$
\lambda_{i}^{(n)} \lambda_{j}^{(n)}=N_{i j}{ }^{k} \lambda_{k}^{(n)}
$$

They are given by the eigenvalues of the matrices $\left(N_{i}\right)_{j}{ }^{k}$.

There exists a remarkable relation between the fusion algebra and the modular transformation $S_{i j}$ as represented on the characters $\chi_{i}$. In words it states that $S$ diagonalizes the algebra. More precisely it expresses the one-dimensional representations $\lambda_{i}^{(j)}$ and the integers $N_{i j k}$ in terms of $S$ as

and

$$
\lambda_{i}^{(j)}=\frac{S_{i j}}{S_{0 j}}
$$

$$
N_{i j k}=\sum_{n} \frac{S_{i n} S_{j n} S_{n k}}{S_{0 n}}
$$

We will prove these relations in the next section.

We can use the result (2.9) to prove that the matrix $S$ is symmetric and unitary, irrespective of whether the 
CFT itself is unitary. The first property follows from the permutation symmetry of the $N_{i j k}$, and is in fact equivalent to it. We can now use in addition the invariance of the fusion algebra under conjugation $C: \phi_{i} \rightarrow \phi_{i}$, which implies $\lambda_{i}^{(j)}=\lambda_{i}^{*(j)}$. Then from (2.8) we find $C S=S^{*}$, which together with $S^{2}=C$ gives unitarity.

For unitary CFT's with positive conformal weights, the quantities $\lambda_{i}^{(0)}$ have a special interpretation. They indicate the relative dimension of the representation $\left[\phi_{i}\right]$ compared to identity representation [1]. Hereto we use the fact that in the limit $q \rightarrow 1$ the character $\chi_{i}(q)$ formally counts the number of states in the representation $\left[\phi_{i}\right]$. More precisely we have

$\frac{\operatorname{tr}_{\left[\phi_{i}\right]}(1)}{\operatorname{tr}_{[1]}(1)}=\lim _{q \rightarrow 1} \frac{\chi_{i}(q)}{\chi_{0}(q)}=\lim _{q \rightarrow 0} \frac{S_{i j} \chi_{j}(q)}{S_{0 k} \chi_{k}(q)}=\frac{S_{i 0}}{S_{00}}=\lambda_{i}^{(0)}$

From this we conclude that $\lambda_{i}^{(0)}>0$. The relative dimensions, which are not necessarily integer, satisfy the fusion algebra, and hence we even have $\lambda_{i}^{(0)} \geq 1$, where equality holds iff $\phi_{i} \times \phi_{\hat{i}}=1$. We will make use of this remark later on.

Another important application of the relation (2.9) is that it gives restrictions on the possible values $c$ and $h$ for a given fusion algebra. This is discussed in [1]. In subsequent sections we will discuss some other applications.

\section{The Proof.}

The proof of the relations (2.8-9) is based on the construction of a set of operators $\phi_{i}(a)$ and $\phi_{i}(b)$ acting on the characters, where $a$ and $b$ are a basis of cycles on the torus. The definition of

$$
\phi_{i}(c): \quad \chi_{j} \rightarrow \phi_{i}(c) \chi_{j}
$$

where $c=a$ or $b$, is in words as follows [1]. One inserts the identity operator inside the trace over $\left[\phi_{j}\right]$ at some point on the torus, and rewrites it as the OPE of an operator in the representation $\left[\phi_{i}\right]$ and its conjugate. Next one moves this operator around the cycle $c$, and when its returned to its original position, one takes again the OPE with the conjugate operator to reobtain the identity. This manipulation defines a linear operator on the space of characters, and is, upto normalization, equal to $\phi_{i}(c)$. By applying the operation $\phi_{i}(b)$ to the character $\chi_{0}$ of the identity, one finds that the representation [1] changes into $\left[\phi_{i}\right]$. We use this fact to fix the normalization of the operators $\phi_{i}(c)$ by the condition:

$$
\phi_{i}(b) \chi_{0}=\chi_{i}
$$

The key equation is that, with this normalization, the operator $\phi_{i}(b)$ acts on the other characters as

$$
\phi_{i}(b) \chi_{j}=N_{i j}^{k} \chi_{k}
$$

Intuitively an equation of this form can be expected, but the integrality of the coefficients is not obvious from the definition of $\phi_{i}(b)$.

Before we discuss the derivation of (3.3), we first like to show how this equation is used to prove the relations (2.9). First we observe that, when we move an operator with representation $\left[\phi_{i}\right]$ around the $a$-cycle, the representation $\left[\phi_{j}\right]$, over which the trace is taken, does not change. Consequently the characters $\chi_{j}$ are eigenstates of the operators $\phi_{i}(a)$.

$$
\phi_{i}(a) \chi_{j}=\lambda_{i}^{(j)} \chi_{j}
$$

Next we consider what happens when we perform the modular transformation $S$. Since $S$ interchanges the $a$ and $b$-cycle, the operator $\phi_{i}(b)$ is, by conjugating with the matrix $S$, mapped onto $\phi_{i}(a)$. By comparing (3.3) and (3.4) we immediately conclude that the modular transformation $S$ diagonalizes the coefficients $N_{i j k}$. Finally, the condition (3.2) enables us to determine the 
eigenvalues $\lambda_{i}^{(j)}$, and express everything in terms of the entries of the matrix $S$. The resulting expressions are given in (2.9).

For the derivation of (3.3) we need to examine the definition described above somewhat closer. In order to avoid proliferation of indices we concentrate on one coefficient $N_{i j k}$ for fixed $i, j, k$. Also, for convenience, we restrict to self-conjugate fields, but the proof is easily generalized to include complex representations. The operator $\phi_{i}(b)$ is defined as a sequence of manipulations on the chiral blocks of the two-point function on the torus. By representing the blocks as $\varphi^{3}$-diagrams these manipulations can be depicted as follows

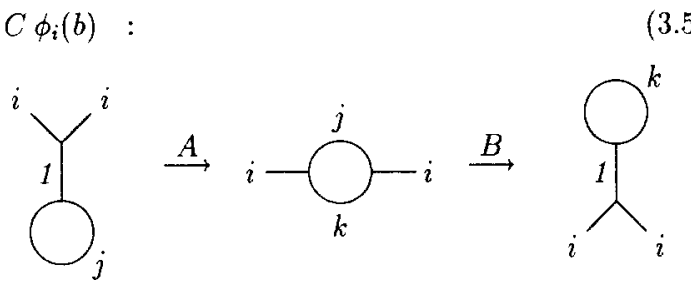

where the normalization constant $C$ is determined by (3.2). (Note that the first and the last diagram represent different markings of the two-punctured torus.) The fact that the operations $A$ and $B$ involve only a part of the $\varphi^{3}$-diagram, allows us to give a realization of the operator $\phi_{i}(b)$ in terms of duality properties of fourpoint functions. We introduce coefficients $A_{\alpha \beta}, B_{\alpha \beta}$ and $C$ defined by the following duality transformations

$A$ :<smiles>IC(I)C(I)I</smiles>

$B$ :<smiles>CC(I)C(C)I</smiles><smiles>CC(C)C(I)C(C)I</smiles><smiles>[3H]C([13CH3])C(I)C(I)I</smiles>

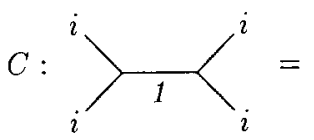

C

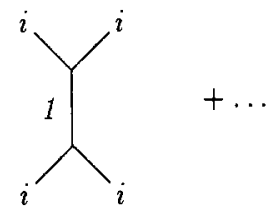

where, $\alpha, \beta=1, . ., N_{i j k}$ label the possible couplings of $i, j, k$. These coefficients depend of course on $i, j, k$ but this is suppressed in the notation. The dots represent terms involving other representations and are not important for us. These terms are dropped in (3.5) and, consequently, the manipulations $A, B$ and $C$ are not invertible. From (3.5) we see that, in order to prove (3.3), we have to show that:

$$
\sum_{\alpha, \beta=1}^{N_{i, k}} \frac{A_{\alpha \beta} B_{\alpha \beta}}{C}=N_{i j k}
$$

The fact that the l.h.s. gives an explicit realization of the operation $\phi_{i}(b)$ was noted independently by several groups $[12,5,6]$. At first sight we don't seem to have gained much, because in general we don't know what the $A, B$ and $C$ coefficients are. However, as Moore and Seiberg showed [5], one doesn't have to know the precise values of these coefficients to derive (3.6). They discovered that a special case of the socalled 'pentagon' identity relates $A, B$ and $C$ and precisely does the job. We will present here a slightly modified version of their proof.

Since for our purpose we don't need the most general case of the pentagon identity, we will apply it directly to a special situation. We consider a sequence of $5 \mathrm{ma}$ nipulations on the chiral blocks of the 3-point function on the torus, with external representations $i, j$ and $k$. We have represented them diagrammatically in the following figure. 


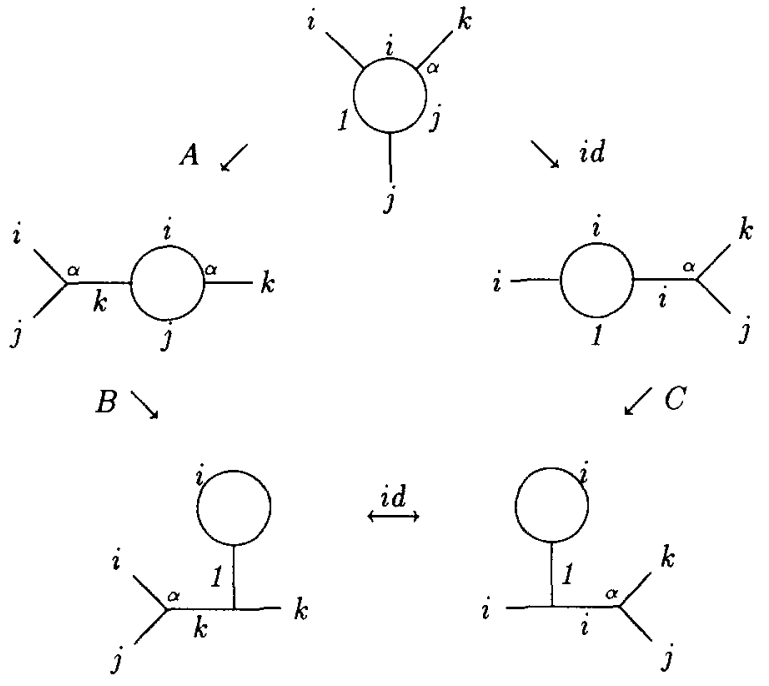

In some diagrams one of the internal representations corresponds to the identity operator. As a result, two of the manipulations are trivial, i.e. they act as the identity map on the chiral blocks. The other three operations are precisely the ones we are interested in, namely $A, B$ and $C$. The direction in which they act is indicated in the figure. Now we observe that there are two ways to get from the top of the pentagon to one of the bottom corners, say the left. One is by applying $A$ and $B$, and the other involves $\mathrm{C}$. The pentagon identity is obtained by requiring that the coefficient one picks up is the same for both choices. It gives the following relation:

$$
\sum_{\beta=1}^{N_{i j k}} A_{\alpha \beta} B_{\alpha \beta}=C
$$

Note that the unlabelled vertex of type $(i j k)$ in the diagram gives rise to the summation over $\beta$, while there is no sum over the coupling $\alpha$. Furthermore $C$ is independent of $\alpha$, so by summing over this coupling $\alpha=$ $1, . ., N_{i j k}$ and dividing by $C$, one derives the desired result (3.6). This concludes our proof of the relations (2.8$9)$.

\section{An Illustration: the 4 State Potts Model.}

The relation between the fusion algebras and the modular properties of the 1-loop characters allows us to extract the fusion rules directly from the holomorphic structure of the partition function. The general method works for any any rational CFT, but will be illustrated with a concrete example. For this we have chosen the 4 state Potts model.

The continuum limit of the 4 state Potts model at criticality has central charge $c=1$. Its chiral symmetry algebra is best understood by representing the model as a $\mathbf{D}_{2}$ orbifold of the $S U(2)_{k=1}$ WZW-model. The discrete group $\mathbf{D}_{2}$ acts on the $S U(2)$ currents $j_{a}(z)$ as

$$
r_{b}: j_{a} \longrightarrow \epsilon_{a b} j_{a}
$$

where the symbol $\epsilon_{a b}$ is defined to be 1 if $a=b$, and -1 otherwise. Accordingly the relevant chiral algebra of this model will be generated by polynomials in the currents invariant under the transformations $r_{a}$. This includes in particular the stress-energy tensor.

The partition function can be written as [13]

$$
Z_{4 s t . P o t t s}=\frac{3}{2} Z_{2}-\frac{1}{2} Z_{1}
$$

where $Z_{N}$ is the partition function of the gaussian model, describing a free scalar field compactified on a circle of radius $N \sqrt{2}$,

$$
Z_{N}(\tau, \bar{\tau})=\sum_{n, m \in \mathbf{Z}} \frac{1}{|\eta(\tau)|^{2}} q^{\left(\frac{n}{N}+m N\right)^{2}} \bar{q}^{\left(\frac{n}{N}-m N\right)^{2}}
$$

In the form (4.2) the holomorphic structure of the partition function is completely obscure. In order to determine the characters of the chiral algebra we introduce the following expressions

$$
\vartheta\left[\begin{array}{l}
\alpha \\
\beta
\end{array}\right]=e^{-2 \pi i \alpha \beta} \frac{1}{\eta(\tau)} \vartheta\left[\begin{array}{l}
\alpha \\
\beta
\end{array}\right](0 \mid 2 \tau)
$$

with $\alpha \in \frac{1}{2 N} \mathbf{Z}_{2 N}, \beta \in \frac{1}{N} \mathbf{Z}_{N}$. The partition function can be expressed in these $\vartheta$-functions, which in turn can 
be combined into characters with a $q$-expansions with integer coefficients. In this way we find the following characters. (We use the same notation for the characters and the operators.)

$$
\begin{aligned}
& 1=\frac{1}{4} \vartheta\left[\begin{array}{l}
0 \\
0
\end{array}\right]+\frac{3}{4} \vartheta\left[\begin{array}{c}
0 \\
1 / 2
\end{array}\right] \\
& h=0 \\
& \sigma_{a}=\frac{1}{2} \vartheta\left[\begin{array}{c}
1 / 4 \\
0
\end{array}\right]+\frac{1}{2} \vartheta\left[\begin{array}{l}
1 / 4 \\
1 / 2
\end{array}\right] \quad(a=1,2,3) \\
& h=\frac{1}{16} \\
& \phi=\frac{1}{2} \vartheta\left[\begin{array}{c}
1 / 2 \\
0
\end{array}\right] \\
& \tau_{a}=\frac{1}{2} \vartheta\left[\begin{array}{c}
1 / 4 \\
0
\end{array}\right]-\frac{1}{2} \vartheta\left[\begin{array}{c}
1 / 4 \\
1 / 2
\end{array}\right] \quad(a=1,2,3) \\
& h=\frac{1}{4} \\
& j_{a}=\frac{1}{4} \vartheta\left[\begin{array}{l}
0 \\
0
\end{array}\right]-\frac{1}{4} \vartheta\left[\begin{array}{c}
0 \\
1 / 2
\end{array}\right] \quad(a=1,2,3) \\
& h=\frac{9}{16} \\
& h=1
\end{aligned}
$$

Note that there are 3 representations $\left[\sigma_{a}\right],\left[\tau_{a}\right]$ and $\left[j_{a}\right]$, $a=1, . ., 3$. This follows from the fact that the corresponding $\vartheta$-function combinations occur with multiplicity 3 in the partition function. So, although only 5 different combinations occur, we have to distinguish 11 independent representations.

From the modular properties of the blocks $v\left[\begin{array}{l}\alpha \\ \beta\end{array}\right]$ we can calculate the behaviour of the characters under the modular transformation $S$. Upto an overall factor $\frac{1}{\sqrt{32}}$ we find the following matrix $S$

\begin{tabular}{|c|ccccc|}
\hline & 1 & $\sigma_{b}$ & $\phi$ & $\tau_{b}$ & $j_{b}$ \\
\hline 1 & 1 & 2 & 2 & 2 & 1 \\
$\sigma_{a}$ & 2 & $\delta_{a b} \sqrt{8}$ & 0 & $-\delta_{a b} \sqrt{8}$ & $2 \epsilon_{a b}$ \\
$\phi$ & 2 & 0 & -4 & 0 & 2 \\
$\tau_{a}$ & 2 & $-\delta_{a b} \sqrt{8}$ & 0 & $\delta_{a b} \sqrt{8}$ & $2 \epsilon_{a b}$ \\
$j_{a}$ & 1 & $2 \epsilon_{a b}$ & 2 & $2 \epsilon_{a b}$ & 1 \\
\hline
\end{tabular}

Note that $S$ is indeed symmetric and unitary. However, if we had identified for example the three $\sigma$-representations this would not be the case. It is easily verified that this matrix satisfies $S^{2}=1$, and consequently all representations are self-conjugate.

By applying (2.7-9) we can read off from the table (4.5) what the fusion algebra of the 4 state Potts model is. We find

$$
\begin{aligned}
\sigma_{a} \times \sigma_{a} & =\tau_{a} \times \tau_{a}=1+\phi+j_{a}, \\
\phi \times \phi & =1+\sum_{c} j_{c}, \\
j_{a} \times j_{a} & =1, \\
\sigma_{a} \times \tau_{a} & =\phi+\sum_{c \neq a} j_{c}, \\
\sigma_{a} \times \sigma_{b} & =\tau_{a} \times \tau_{b}=\sigma_{a} \times \tau_{b}=\sigma_{c}+\tau_{c}, \\
j_{a} \times j_{b} & =j_{c}, \quad a \neq b \neq c .
\end{aligned}
$$

Other fusion rules from cyclicity of the $N_{i j k}$. The interpretation of these fusion rules, similar results for other $c=1$ models and the generalization to other orbifold models are deferred to [10].

As a final remark, we give here the number of generalized characters for the 4 state Potts model on a genus $g$ surface. As explained in [1] this number can be obtained from the first column (or row) of the matrix $S$. We get

$$
\sum_{j}\left(S_{0 j}\right)^{2-2 g}=8^{g-1}\left(4^{g}+7\right),
$$

which is an integer as it should be.

\section{Off-diagonal Invariants and Algebra}

\section{Automorphisms}

The fusion algebra is clearly related to the selection rules for the operator algebra of the primary fields $\Phi_{i \vec{i}}$. However, these are not really one and the same thing, since the fusion algebra, as we define it, concerns only one chiral half of the CFT. In fact, one can associate two fusion algebras with a CFT, one for the left and one for the right representations; the primary fields $\Phi_{i i}$ feel both of them. The coefficients $N_{i j k}$ and $N_{\overline{i j k}}$ give us information about the left and right chiral blocks, which have to be combined in a consistent way to build the correlation functions. We therefore expect some relation 
between $N_{i j k}, N_{\overline{i j k}}$ and the allowed representations $(i, \bar{i})$ of the primary fields.

First let us consider what modular invariance tells us. By writing the partition function as $Z=\bar{\chi} \cdot \Pi \cdot \chi$, we define a matrix $\Pi_{i j}$, with non-negative integer entries. Full modular invariance of the partition function gives two conditions. First the spins $h_{i}-h_{\bar{i}}$ are required to be integer if $\Pi_{i \bar{i}} \neq 0$. Furthermore, invariance under $\tau \rightarrow-1 / \tau$ tells us that $S^{\dagger} \Pi S=\Pi$, which is, since $S$ is unitary, equivalent to

$$
S \Pi=\Pi S
$$

Note that for every chiral algebra there always exists a diagonal modular invariant with $\Pi_{i j}=\delta_{i j}$. However, in this section we are mainly interested in the off-diagonal invariants, because most of the things we are about to discuss are more or less trivial in the diagonal case.

For the following it is important that we consider the maximal chiral symmetry algebra of the model. In other words, we assume that all chiral operators are contained in the identity representation $\left[\phi_{0}\right]=[1]$. This can be stated as

$$
\Pi_{i 0}=\Pi_{0 i}=\delta_{i 0}
$$

We now like to show that (5.1) and (5.2) imply that each representation $\left[\phi_{i}\right]$ occurs precisely once and is uniquely paired with a representation $\left[\bar{\phi}_{i}\right]$ of the right algebra. More precisely, we will prove that the integral matrix II defines a one to one mapping (=permutation) of the representations

$$
\text { II : } \phi_{i} \rightarrow \phi_{\bar{i}},
$$

and furthermore that this mapping gives an automorphism of the fusion algebra. Our proof works only for unitary CFT's, but we believe that these facts also hold for non-unitary models.

We first consider the relative dimensions defined in
(2.10). Combining the relations (5.1) and (5.2) gives

$$
\lambda_{i}^{(0)}=\Pi_{i j} \lambda_{j}^{(0)}
$$

Now we make use of the observation that for unitary CFT's all relative dimensions satisfy $\lambda_{i}^{(0)} \geq 1$. Since $\Pi$ has non-negative integer entries, equation (5.4) clearly implies that for each representation $i$ there is one and precisely one representation $\bar{i}$ such that $\Pi_{i j}=\delta_{i j}$, and hence $\Pi$ is indeed of the form (5.3).

Next we consider the interplay between this mapping and the left and right fusion algebras. Using the condition (5.1) together with (5.4) one easily shows that for the one-dimensional representations one has: $\lambda_{\vec{i}}^{(j)}=$ $\lambda_{i}^{(\bar{j})}$. This in turn is sufficient to prove that the integer fusion coefficients satisfy

$$
N_{\overline{i j k}}=N_{i j k}
$$

which shows that (5.3) is an automorphism of the fusion algebra

Another immediate consequence of (5.5) is that for any correlation function of the primary fields $\Phi_{i \vec{i}}$ the numbers of left and right chiral blocks are equal. This suggests that also in this more general case the correlator defines a one-to one pairing between the chiral blocks, such that corresponding blocks transform in isomorphic representations of the modular group. In particular, by considering the three-point function of the primary fields we may conclude that the selection rules for the OPA of the primary fields $\Phi_{i i}$ are determined by only one of the labels $i$ or $\bar{i}$. Consequently, these selection rules have the same form as the fusion algebra. For off-diagonal CFT's this a non-trivial fact, which follows from the modular invariance of the one-loop partition function, and is some cases even equivalent to it. 


\section{Some Further Examples}

To illustrate the relation between automorphisms of the fusion algebra and off-diagonal modular invariant partition functions we will consider the level $k S U(2)$ WZW-models. As is well known the $S U(2)_{k}$ Kac-Moody algebra allows for $k+1$ integrable representations $\left[\phi_{j}\right]$ $(2 j=0,1, \ldots, k)$ with $S U(2)$ isospin $j[14]$. The weights of the corresponding primary fields are given by $h_{j}=$ $j(j+1) /(k+2)$. The characters will be denoted as $\chi_{\lambda}$ with $\lambda=2 j+1$. We will freely make use of both labels $j$ and $\lambda$.

The investigation of the modular invariant partition functions that can be constructed with these $S U(2)_{k^{-}}$ characters $\chi_{\lambda}$ has yielded the famous $A D E$ classsification of Cappeli, Itzykson and Zuber [15]: all modular invariant combinations can be labelled by the Dynkin diagrams of the simply laced Lie algebras. The $A_{n}$ series corresponds to the diagonal sum over all representations. The $D_{\text {even }}, E_{6}$, and $E_{8}$ models are diagonal combinations with an extended chiral algebra, which in the latter two cases has been identified with the $C_{2}$ respectively the $G_{2}$ level 1 Kac-Moody algebras [16]. The $D_{\text {odd }}$ series, and $E_{7}$ are off-diagonal combinations and will be discussed below.

The $D_{2 n+1}$ combinations occur at level $k=4 n-2$. In the partition function the same characters occur as in the corresponding $A_{n}$ form, only the pairing is not diagonal. More precisely in these models the left and right representation $\left[\phi_{j}\right]$ and $\left[\bar{\phi}_{\bar{j}}\right]$ are paired as

$$
\begin{array}{ll}
\bar{j}=\frac{1}{2} k-j, & j \in \mathbf{Z}+\frac{1}{2}, \\
\bar{j}=j, & j \in \mathbf{Z} .
\end{array}
$$

The fusion rules are of course identical to those of the $A_{n}$ series, since they only depend on the chiral algebra. For the $S U(2)_{k}$ Kac-Moody algebra the fusion algebra is given by

$$
\phi_{j} \times \phi_{j^{\prime}}=\sum_{j^{\prime \prime}=\left|j-j^{\prime}\right|}^{\min \left(j+j^{\prime}, k-j-j^{\prime}\right)} \phi_{j^{\prime \prime}}
$$

with $j^{\prime \prime}-\left|j+j^{\prime}\right| \in \mathbf{Z}$. These fusion rules can be obtained by analyzing the differential equations for the correlators [14] or by using the modular properties of the characters $\chi_{\lambda}$. The matrix $S_{\lambda \lambda^{\prime}}$ reads

$$
S_{\lambda \lambda^{\prime}}=\left(\frac{2}{k+2}\right)^{\frac{1}{2}} \sin \frac{\lambda \lambda^{\prime}}{k+2} \pi
$$

and by plugging this into (2.9) it is not difficult to derive $(6.2)$.

Let us now verify that the transformation (6.1) constitutes an automorphism of the fusion rules (6.2). The case $j$ and $j^{\prime}$ both integer is more or less trivial, since they do not produce half-integer $j^{\prime \prime}$. Also when $j$ and $j^{\prime}$ are both half-integer it is easily verified that everything works. In the remaining case, i.e. if $j \in \mathbf{Z}$ and $j^{\prime} \in \mathbf{Z}+\frac{1}{2}$ or vice versa, one needs the relation

$$
\min \left(j+j^{\prime}, k-j-j^{\prime}\right)=\frac{1}{2} k-\left|\frac{1}{2} k-j-j^{\prime}\right| .
$$

to check that the r.h.s. of $(6.2)$ is invariant under $j^{\prime} \rightarrow$ $\frac{1}{2} k-j^{\prime} j^{\prime \prime} \rightarrow \frac{1}{2} k-j^{\prime \prime}$. This shows that (6.1) is indeed an automorphism of the fusion algebra (6.2). It is not difficult to see that there are no other automorphisms of the fusion algebra (6.2). Note that we only needed that $k$ was even. The fact that the $D_{2 n+1}$ invariants only occur at $k=4 n-2$ follows from the condition $h_{j}=h_{\tilde{j}} \bmod 1$.

A more peculiar example is the $E_{7}$ off-diagonal modular invariant. The corresponding diagonal invariant is the one labelled by $D_{10}$. Their partition functions are given by

$$
\begin{gathered}
D_{10}: Z=\left|\chi_{1}+\chi_{17}\right|^{2}+\left|\chi_{3}+\chi_{15}\right|^{2}+\left|\chi_{5}+\chi_{13}\right|^{2} \\
+\left|\chi_{7}+\chi_{11}\right|^{2}+2\left|\chi_{9}\right|^{2} \\
E_{7}: Z=\left|\chi_{1}+\chi_{17}\right|^{2}+\left|\chi_{5}+\chi_{13}\right|^{2}+\left|\chi_{7}+\chi_{11}\right|^{2} \\
+\left|\chi_{9}\right|^{2}+\left[\left(\chi_{3}+\chi_{15}\right) \bar{\chi}_{9}+\text { c.c. }\right]
\end{gathered}
$$


First we see that in both models the character $\chi_{9}$ occurs twice, and accordingly two different fields with $j=4$ have to be distinguished. We shall label the characters as $\chi_{9}^{+}$and $\chi_{9}^{-}$. Both the $D_{10}$ and $E_{7}$ combinations have the same chiral algebra, which contains the $S U(2)_{k=16}$ KM-algebra. The characters are given by $\chi_{1}+\chi_{17}, \chi_{3}+$ $\chi_{15}, \chi_{5}+\chi_{13}, \chi_{7}+\chi_{11}, \chi_{9}^{+}$and $\chi_{9}^{-}$. The corresponding operators will be denoted as $1, \phi_{1}, \phi_{2}, \phi_{3}, \phi_{4}^{+}$, and $\phi_{4}^{-}$. The off-diagonal $E_{7}$ pairing is given by $\phi_{1} \leftrightarrow \phi_{4}^{+}$.

Since both models have the same chiral algebra, the fusion rules will be identical. We will derive them from the modular behaviour of the characters. The matrix $S$ is most easily obtained by folding up the $S U(2)_{k=16}$ matrix and requiring $S^{2}=1$. The result reads upto normalization,

\begin{tabular}{|c|ccc|}
\hline & $\lambda^{\prime}$ & $9^{+}$ & $9^{-}$ \\
\hline$\lambda$ & $2 s_{\lambda \lambda^{\prime}}$ & $(-1)^{j}$ & $(-1)^{j}$ \\
$9^{+}$ & $(-1)^{j^{\prime}}$ & 2 & -1 \\
$9^{-}$ & $(-1)^{j^{\prime}}$ & -1 & 2 \\
\hline
\end{tabular}

with $s_{\lambda \lambda^{\prime}}=\sin \frac{\lambda \lambda^{\prime}}{18} \pi$. From this we get the following set of fusion rules for these representations

$$
\begin{aligned}
\phi_{1} \times \phi_{1} & =1+\phi_{1}+\phi_{2} \\
\phi_{1} \times \phi_{2} & =\phi_{1}+\phi_{2}+\phi_{3} \\
\phi_{1} \times \phi_{3} & =\phi_{2}+\phi_{3}+\phi_{4}^{+}+\phi_{4}^{-} \\
\phi_{1} \times \phi_{4}^{+} & =\phi_{3}+\phi_{4}^{+} \\
\phi_{4}^{+} \times \phi_{4}^{+} & =1+\phi_{2}+\phi_{4}^{+} \\
\phi_{2} \times \phi_{4}^{+} & =\phi_{2}+\phi_{3}+\phi_{4}^{+} \\
\phi_{3} \times \phi_{4}^{+} & =\phi_{1}+\phi_{2}+\phi_{3}+\phi_{4}^{-} \\
\phi_{4}^{+} \times \phi_{4}^{-} & =\phi_{1}+\phi_{3} \\
\phi_{2} \times \phi_{2} & =1+\phi_{1}+\phi_{2}+\phi_{3}+\phi_{4}^{+}+\phi_{4}^{-} \\
\phi_{3} \times \phi_{3} & =1+\phi_{1}+2 \phi_{2}+2 \phi_{3}+\phi_{4}^{+}+\phi_{4}^{-} \\
\phi_{2} \times \phi_{3} & =\phi_{1}+\phi_{2}+2 \phi_{3}+\phi_{4}^{+}+\phi_{4}^{-}
\end{aligned}
$$

It is readily verified that the $E_{7}$ pairing $\phi_{1} \leftrightarrow \phi_{4}^{+}$is indeed a symmetry of these fusion rules. It is also not so hard to convince oneself that this automorphism does not generalize to the whole $D_{\text {even }}$ series, and that $D_{10}$ is in fact the only possibility.

\section{Acknowledgements}

We acknowledge useful discussions with R. Brustein, C. Vafa, and H. Verlinde.

\section{References}

[1] E. Verlinde, Nucl. Phys. B300 (1988) 360.

[2] C. Vafa, Phys.Lett. 206B (1988) 421.

[3] K.-H. Rehren and B. Schroer, Einstein Causality and Artin Braids, Berlin-preprint (April 1988).

[4] S. Mathur, S. Mukhi, and A. Sen, Differential equations for correlators and characters in arbitrary rational conformal field theories, preprint TIFR/TH/88-32.

[5] G. Moore and N. Seiberg, Polynomial Equations for Rational Conformal Field Theories, preprint IASSNS-HEP 88/18.

[6] R. Brustein, S. Yankielowicz, and J.-B. Zuber, Factorization and Selection Rules of Operator Product Algebras in Conformal Field Theories, preprint SPhT/88-086 TAHP-1647-88.

[7] D. Friedan and S.H. Shenker, Nucl.Phys. B281 (1987) 509.

[8] J.L. Cardy, Nucl.Phys. B270 [FS16] (1986) 186.

[9] A.A. Belavin, A.M. Polyakov, and A.B. Zamolodchikov, Nucl.Phys. B241 (1984) 333. 
[10] R. Dijkgraaf, C. Vafa, E. Verlinde and H. Verlinde, in preparation.

[11] V. Kac and M. Wakamoto, Modular and conformal invariance constraints in representation theory of affine algebras, M.I.T.-preprint.

[12] E. Verlinde, H. Verlinde, unpublished.

[13] R. Dijkgraaf, E. Verlinde, and H. Verlinde, Comm. Math. Phys. 115 (1988) 649.
[14] D. Gepner and E. Witten, Nucl.Phys. B278 (1986) 493.

[15] A. Capelli, C. Itzykson, and J.-B. Zuber, Nucl. Phys. B280 [FS18] (1987) 445; Comm.Math.Phys. 113 (1987) 1; D. Gepner, Nucl.Phys. B287 (1987) 111.

[16] P. Bouwknegt and W. Nahm, Phys. Lett. 184B (1987) 359. 